\title{
Information-aware access network selection
}

\author{
Konstantinos V. Katsaros \\ Intracom Telecom \\ konkat@intracom-telecom.com
}

\author{
Denis Shtefan, Ioannis Psaras, Vasilis \\ Sourlas, Sergi Rene, George Pavlou \\ University College London \\ \{denis.shtefan.13, i.psaras,v.sourlas, \\ s.rene,g.pavlou\}@ucl.ac.uk
}

\begin{abstract}
Mobile devices are increasingly presented with multiple connectivity options, including WiFi hotspots, micro-/macrocells or even other devices in device-to-device communications. In this environment, connectivity management has been focused on contention, congestion and wireless medium conditions. In this paper, we assess the role of informationcentrism in mobile device connectivity management. Motivated by the increasing availability of content and services in in-network caches and micro-data centers, we design an access network selection scheme that takes into account information availability within each connectivity option. Using extensive simulations, we show that information-awareness results in a significant increase of experienced cache hit ratios, i.e., up to $115 \%$ in certain scenarios.
\end{abstract}

\section{Keywords}

information-centric networking, wireless, WiFi, HetNets, offloading.

\section{INTRODUCTION}

The number of connectivity points in urban environments has increased significantly in recent years. Mobile devices already have got tens of connectivity options at any given moment, mainly in the form of $\mathrm{WiFi}$ access points [3], but are also expected to be presented with even more options with the emergence of the so call Hetnets [4]. Additionally, more than 50 billion devices expected to be connected to an Internet of Things (IoT) by 2020 [11], increasing the access, communication and connectivity points by at least an order of magnitude. In this landscape, a good percentage of those (IoT) devices, and corresponding connectivity points, will be dedicated to application specific purposes (e.g., house temperature management). At the same wide the availability of content in caches and of services in computation spots (in an edge/fog computing realm [6]), observed in the context of rich connectivity environments, will inevitably bring connectivity in the forefront of attention, as connectivity points are defined and linked to the information available in the corresponding network environment.
Building on these observations, we argue that in view of the rich connectivity environments emerging, connectivity options should be linked to information itself. In this paper, we provide a first attempt to associate link-layer connectivity to content discovery/availability, focusing on the current connectivity landscape where multiple connectivity points, such as WiFi APs, provide access to the network and the information residing there in the form of cached or pre-fetched content.

In recent years, there has been a substantial body of work focusing on the opportunities arising in this environment in the form of WiFi offloading, e.g., [2]. The ultimate goal of $\mathrm{WiFi}$ offloading is to relieve the cellular network from increased load. The most prominent solutions in this space focus on pre-fetching content in storage-enabled WiFi APs and serving user requests from those APs. Although complicated redirection solutions exist in the IP space, in an ICN environment much of this functionality is inherently supported. That is, named content can be stored in WiFi AP caches and served from there upon subsequent requests.

However, unless users broadcast/flood their request to all available APs, requests will be directed to one $\mathrm{AP}$ and from there they will head towards the origin source. In more detail, there is a salient point pertaining to all ICN architectural proposals: requests or Interests are always forwarded towards the original content source. Hence, in case content exists in some neighbouring AP, even an ICN mode of operation will not be able to discover existing copies of requested content. Although some alternative proposals have started to appear (e.g., $[19,16])$, there is no forwarding framework to redirect requests downwards towards the edge of the network in any of the mainstream architectures. What is more important, even with such proposals integrated within some ICN architecture, paths are likely to be disjoint, given that APs might well belong to different ISPs. In these cases, the available information cannot be reached.

It therefore becomes apparent, that in order to enable access to all potentially available content/information in a (wireless/mobile) networking environment, the main 
request-response functionality of ICN designs should be coupled by the support of information-aware network selection at the link layer. Though substantial work has been devoted to the design of intelligent offloading and vertical handover mechanism [2, 22], the resulting solutions are information-agnostic, focusing on the (wireless) network conditions.

The contributions of this paper are as follows:

- We propose and investigate the concept of Information-Centric Connectivity (ICCON) in the particular context of access-network selection and WiFi offloading. We note however, that the broader concept proposed in this paper applies to D2D ad hoc communication between mobile devices as well as to Hetnets.

- We introduce a profile matching mechanism, according to which user interests, as well as the available content, are built into profiles, which are then compared to support connectivity decisions.

- We investigate different access network selection modes, subject to the application characteristics.

- We propose two different deployment methods and corresponding control plane mechanisms for the support of the envisioned functionality, where the offloading decision is either controlled by mobile network operators or supported by WiFi network operators.

- We evaluate the performance of the proposed mechanisms, showing that the introduction of information awareness in connectivity management can increase the observed cache hit ratio by even up to $115 \%$ in certain cases.

We note that the mechanisms proposed here do not depend on some specific ICN architecture, nor do they require the prior deployment of any ICN architecture in order to be operational. The basic functionality can work over the TCP/IP protocol stack, although ICN features integrated into the routing architecture would improve its performance further.

The rest of the paper is organized as follows. In Section 2 we present an overview of the proposed access network selection scheme, presenting various different operation modes and deployment options. In Section 3, we elaborate on specific mechanisms for the realization of the proposed functionality, building on currently available technology. We present an extensive performance evaluation study in Section 4. Finally, we discuss related work in Section 5 and conclude in Section 6 , further discussing future work.

\section{OVERVIEW}

\subsection{Networking environment}

In this work we focus on the support of informationaware selection of access networks, focusing on information availability in the form of cached content. However, the presented mechanism can be also applied in the case of in-network services, that is, in cases where user-facing applications run on WiFi APs, eNBs or other access network locations in a Mobile Edge Computing environment [6]. Here we concentrate on WiFi environments, but we note that the concept of "Information-aware network selection" is directly applicable in Hetnet environments in the form of cell selection [4].

For our baseline setup, we consider environments of dense WiFi AP deployments, with each AP advertising a unique Service Set Identifier (SSID). For simplicity, we assume that each AP provides Internet connectivity through a distinct access network $i \in[1 . . M]$, where $M$ is the total number of available networks, though more complex environments can be supported as well, e.g., virtual WLANs. One or more content caches are hosted on APs themselves or other devices further inside the network, e.g., content-centric routers, HTTP caches in middleboxes, etc. We denote each cache as $c_{i j}, i \in[1 . . M], j \in[1 . . K]$, where $K$ is the total number of caches in the particular access network. For simplicity, in the following we assume a fixed $K$ value across all $M$ networks.

Mobile users are interested in content issuing requests through the AP they are connected to each time. These requests are submitted to the network and subsequently forwarded towards the content provider (see also Figure 2(a)). Request forwarding may be accomplished either via means of the current TCP/IP stack, e.g., forwarding of an HTTP request based on the resolved URL-to-IP mapping, or over an ICN-enabled mechanism such as CCN/NDN [14]. As we will see in the following, the envisioned mechanisms can be supported on top of any of these architectures.

\subsection{Frequency of connectivity decisions}

Being presented with multiple connectivity options UEs aim at connecting to the AP that is expected to lead them to the desired content in terms of a cache hit. This expectation is quantified in terms of a fit function $F$, which further takes into account other criteria such as the load on each AP (see Section 3.4). The evaluation of the fit function, and the subsequent selection of an AP can take place at various temporal granularities, subject to the characteristics of the application environment. More specifically, in this work we consider the two following modes of access network selection.

Per request mode. This mode corresponds to applications such as video streaming, where a single content request results in the delivery of a considerable amount of data, during which the user is assumed to stay connected (no mobility). In this case, the selection of an access network is based on whether the desired content item is cached in the network.

Per session mode. In this mode, a single AP selection is made by a UE for the entire time it remains 
at a certain location, during which multiple content requests may be issued. In this case, the selection of an access network is based on an estimation of the content that the UE is expected to request and a subsequent estimation of its availability in the available networks.

Obviously, the per request mode appears as the most promising since it is based on an exact look-up of the desired content item, rather than an estimation. However, the delays imposed by this look-up operation (i.e., transfer of the content identifiers, lookup operation, transfer of the response (see Section 3.3)) may have disproportional effect on the quality of experience for the user when the content retrieval process is expected to be short. We leave the design of a mode selection mechanism for future work.

\subsection{Assessing information availability}

In order to allow the assessment of the potential availability of information within each network, each cache builds a cache profile describing the content currently available. As more than one caches may be available within a network (e.g., the case of hierarchical, cooperative caches), all cache profiles contribute to the creation of the network profile, which is used to assess the availability of the desired information. This assessment is straightforward in the case of the per request selection mode, as it results in a simple look-up operation of an information/content identifier (see Section 3).

In the per session selection mode, the envisioned mechanism builds on an estimation of the information desired by the user. To this end, UEs keep track of the issued requests so as to locally build a user profile describing the user's interests through time.

The objective of the proposed mechanism(s) then, is to enable the comparison of user profiles ${ }^{1}$ against network profiles, so as to identify the corresponding networks with the best matching to user interests, and hence the highest likelihood of a cache hit. To this end, the proposed mechanism(s) supports a series of functional features: (i) the exchange of profiles so as to enable their comparison, (ii) the comparison of the available profiles and the ranking of the available networks wrt. their matching to a UE's interests, (iii) the delivery of the network ranking to the UE and (iv) its subsequent use in selecting and connecting to a candidate network.

\subsection{Deployment model}

The exact realization of these functional features is subject to the deployment model of the overall concept. In this paper, we consider two distinct deployment mod-

\footnotetext{
${ }^{1}$ In order to simplify the description of the proposed mechanisms, in the following we use the term user profile to also refer to unique information identifiers in the case of the per request selection mode, unless otherwise specified.
}

els, with corresponding distinct mechanism realizations. Mobile operator managed mechanism. In this model, the mechanism is operated by a mobile network operator, in the interest of managing and controlling traffic offloading. This model requires cooperation between mobile operators and WiFi operators (if distinct), for the exchange of information regarding the current information availability, as well (wireless) network conditions (see Section 3.3.1).

WiFi operator supported mechanism. In this model, the control of the mechanism is split between WiFi operators and UEs. A WiFi operator may control multiple APs in a networking area, utilizing the proposed mechanism in directing its customers to the most appropriate AP. In another scenario, multiple WiFi operators compete in providing the cached content (and/or services [9]) that best fits the interests of roaming users (see Section 3.3.2).

In the following we provide a detailed description of the basic components of the proposed mechanism(s) including the creation of UE and network profiles and the identified functional features for both of the identified deployment models and access selection modes.

\section{SELECTION MECHANISM}

\subsection{Profiles}

The purpose of the considered user profiles is to reflect the user interests. To this end, and wrt. the per session selection mode (see Section 2.2), locally monitor the content requests resulting from user's actions on the application layer, so as to identify the content requested by the user. As the information space is vast and users may request particular content items only once, i.e., one-timers, this monitoring mechanism further aims to identify the current popularity of each requested content item, wrt. the total population of requests submitted by the user. To this end, a virtual cache is maintained by a UE. That is, content requests submitted by the user are fed to a limited-size $(u)$ cache index which maintains their popularity ordering using a cache replacement policy, e.g., LRU, LFU, etc. Note that the purpose of this cache is not to actually cache the content, but to rather track content popularity in time.

The exact realization of the proposed virtual cache mechanism (including the interception of content requests), depends on the network architecture and the corresponding protocol stack of UEs. In the case of $\mathrm{CCN} / \mathrm{NDN}$ hosts the virtual cache is realized at the network layer which is responsible for the creation and handling of Interest messages, i.e., the requests. In the case of the current TCP/IP protocol stack, we note the proliferation of the HTTP protocol as the means to retrieve content either through browsers or purpose specific (smart) applications. TCP/IP hosts can support 
the proposed virtual cache functionality at a thin layer below the application layer, responsible for retrieving the content identifier (i.e., , the REQUEST-URI) in submitted HTTP requests.

Given the maintained (virtual) cache indexes, in this work we consider Bloom filters [5] for the space efficient and privacy preserving representation of the UE and cache/network profiles. More specifically, for each profile, an $m$ bit Bloom filter is used as a membership set for content identifiers included in the (virtual) cache index. The Bloom filter configuration (i.e., size $(m)$ and set of ( $k$ ) hash functions) is fixed across participating caches, networks and UEs and is specified subject to the considered cache sizes, the number of caches in a certain network (see next) and false positive constraints. Note also that cache profiles may only contain a subset of the available cache indexes (i.e., the top-most popular items) so as to achieve a balance between the efficiency of the mechanism and the size of the profiles ${ }^{2}$. We denote UE profiles with $b_{U E}$ and cache profiles with $b_{c}^{i j}, i \in[1 . . M], j \in[1 . . K]$.

Network profiles $\left(b_{n e t}\right)$ are created based on the profiles of the caches that may serve the UEs connecting to the network. Namely, a network profile is created by applying the binary OR operation on all $b_{c}^{i j}$ profiles in the network. Note that in this process, the set of considered caches is defined by the particular caching architecture of the network, i.e., off-path caches may also be considered in cooperative caching schemes that support redirection of requests.

\subsection{Profile comparison}

The comparison of an UE profile against a network profile is performed by applying the binary AND operation between the two filters. The UE-network profile match function $f$ is defined as the ratio of the total number of bits set to one after the operation, over the total number of one bits in the network profile, i.e., if $b p_{n e t}$ is the total number of ones marked in the network profile $b_{n e t}, b p_{U E}^{A N D}$ is the total number of ones after the AND operation, then we define the UE-network profile match function $f$ as:

$$
f=\frac{b p_{U E}^{A N D}}{b p_{\text {net }}} .
$$

Note that this definition applies in the case of the per session selection mode, where multiple content items are simultaneously looked-up in the network profile. The per request selection method involves a much simpler binary fit function.

\footnotetext{
${ }^{2}$ The lifetime of content items in the caches, as well as the frequency by which the cache profiles are updated, play an important role in the configuration of the Bloom filter sizes. We defer this discussion to future work.
}

$$
f= \begin{cases}1, & \text { if item is available } \\ 0, & \text { otherwise }\end{cases}
$$

\subsection{Profile exchange}

Having established a method for the creation of user and network profiles, the proposed operation further necessitates the availability of mechanisms that bring UE and network profiles together so as to be compared. The exact design of these mechanisms strongly depends on the deployment model.

\subsubsection{Mobile operator managed mechanism}

In this case, the proposed functionality is orchestrated by mobile operators so as to efficiently manage offloading. 3GPP has standardized the Access Network Discovery and Selection Function (ANDSF) for LTE networks, which is responsible for providing UEs with prioritized lists of access technologies / access networks [1]. We therefore consider the ANDSF as the natural location for placing the proposed functionality within mobile networks. As shown in Figure 3.3.1, the ANDSF establishes communication interfaces with both UEs and WiFi operators and/or individual AP/cache devices. On the UE side, UEs submit their profiles to ANDSF periodically over the S14 (TCP/IP) interface [1]. The frequency of this operation depends on the dynamics of users both in terms of mobility, i.e., how frequently they are faced with access network selection decisions, and content interests, i.e., how fast their interests evolve in time.

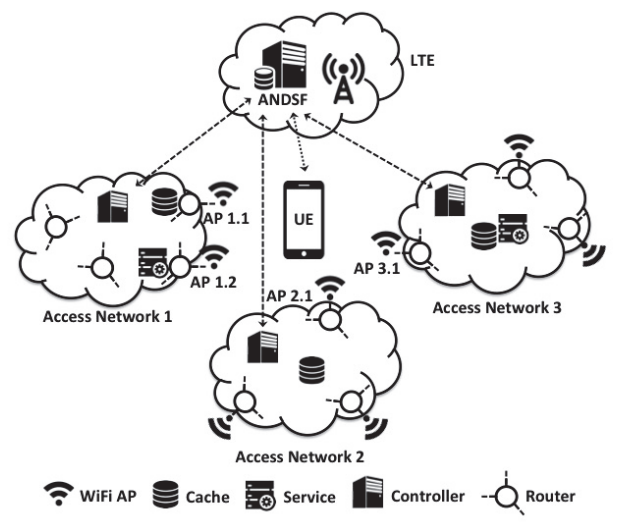

Figure 1: Mobile operator controlled mechanism.

On the fixed part of the network, the ANDSF component collects network profiles by the WiFi operators. This operation assumes the availability of a broader monitoring mechanism responsible for the collection of information related to the overall conditions of the access networks, both on the wireless domain (i.e., AP user load, observed rates, etc.) and the fixed network, e.g., network congestion, cache load, etc. Such mech- 
anisms are already under development for the support of simple, information-agnostic offloading mechanisms, e.g., [13].

UEs either report their geographical position or a list of discovered WLANs in their vicinity to the ANDSF. The ANDSF compares the available UE and network profiles (see Section 3.2) and subsequently ranks the available networks according to a wider set of criteria, e.g., network/AP/cache load (see Section 3.4). The ranked network list is provided to the UE, which then connects to the preferred network.

\subsubsection{WiFi operator supported mechanism}

In this case, the proposed functionality is supported by WiFi operators and aims at enabling localized decisions made by UE's themselves. UEs autonomously interact with APs to collect network profiles and locally compare them against their own profiles. In order to avoid the delay and energy overheads associated with the sequential association of a UE with each candidate AP for collection of network profile information, we consider a link layer mechanism for this purpose.

The Generic Advertisement Service (GAS) of IEEE $802.11 u$ [12] provides a generic frame format and exchange process enabling UEs to query APs for particular information. The Access Network Query Protocol (ANQP) defined in IEEE 802.11u, is a particular protocol employing GAS, already widely used for the delivery of authentication configuration, load and other information, to UEs. Within ANQP, the vendor-specific list has been foreseen as a generic container for vendor information. We consider this field to allow APs to convey network profiles to UEs at the link layer. In an alternative approach, the recently announced $\mathrm{WiFi}$ Neighbour Awareness Networking (NAN) protocol [8] also further supports a low energy consumption device discovery mechanism enhanced with publish/subscribe primitives that can be employed for the same purpose.

Based on the retrieved network profiles, UEs are in the position to assess the available content in each available network (see Section 3.2). As in the case of the mobile operator mechanism, the final network selection is subject to a wider set of criteria (see Section 3.4), based on additional information provided by APs through the same ANQP-based mechanism.

Even though the described operation avoids the time consuming sequential association with the available APs, considerable delay overheads may still be incurred by the delivery of the network profiles, especially when their size is tailored to support large cache indexes. For this reason, this mechanism is considered more suitable for the lightweight per request selection method. In this case, the GAS Request/Response Frames can be used to deliver the (encrypted) content identifier the user is interested in, as well as the response from the AP. Sim- ilar mechanisms for the exchange of content identifiers have been also proposed in [21].

\subsection{Access network selection}

The final selection of an access network is subject to a wide range of criteria which have extensively studied in the past, both in the context of vertical handover decisions [22] and offloading mechanisms [2]. Though this work focuses on information availability as a new criterion for network selection, we acknowledge the importance of performance related metrics such as the received signal strength, downlink/uplink throughput and signal-to-noise ratio, as well as other aspects, such as monetary costs and security parameters (e.g., encryption). In this context, access network selection in this work is based on a fit function $F$ that balances between UE-network profile match $(f)$ and other criteria. In order to focus on the investigation of information availability as the newly introduced criterion, we consider a simplified fit function $F$ that takes into account the weighted $(W)$ average of $f$ and the $\operatorname{AP}$ load $(l)$, i.e.,

$$
F=W f+(1-W) l,
$$

and $l$ is calculated as:

$$
l=1-\frac{n_{i}}{\left(\sum_{i=1}^{M} n_{i}\right)},
$$

where $n_{i}$ denotes the number of users currently being served at $\operatorname{AP} i, i \in[1 . . M]$.

\section{PERFORMANCE EVALUATION}

We next engage in a detailed investigation of the performance of the proposed schemes, with the objective to quantify the potential benefits stemming from the introduction of information-awareness as an access network selection criterion. To the best of our knowledge, this is the first study of the effects of information-awareness in access network selection, and as such, it does not aim at an exhaustive study of other criteria well investigated in the past (see Section 3.4).

\subsection{Framework}

Simulator. For our evaluation purposes we implemented a simulation framework adopting the $\mathrm{CCN} / \mathrm{NDN}$ architecture [14]. Our simulation model is based on the CCNSim [17] chunk level simulator, build for the OMNeT ++ simulation framework [20]. Focusing on the introduction of information-awareness, our simulation model reflects a simplified approach where we omit the details of the wireless environment, e.g., signalstrength, interference, etc., as well as medium access control (MAC) aspects (e.g., contention).

Network model. We consider a network environment in which a set of $N=150 \mathrm{UEs}$, affiliated with a single mobile operator, visit a certain location, e.g., a shopping mall or an airport lounge, where a set of $M=10$ 


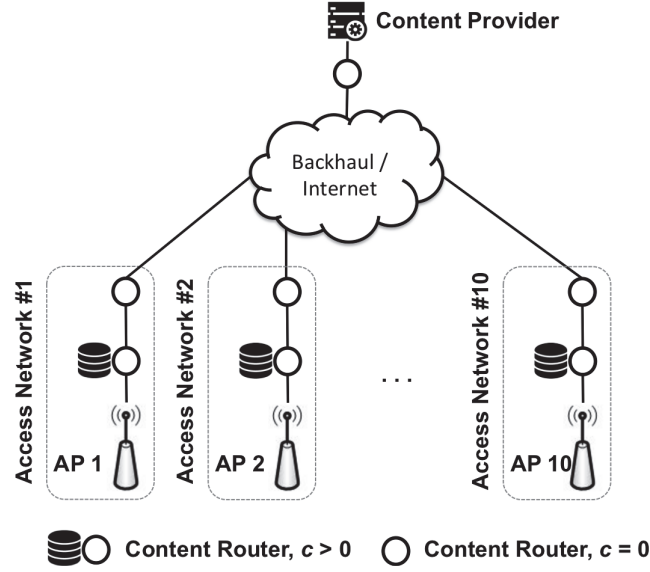

(a) Access networks

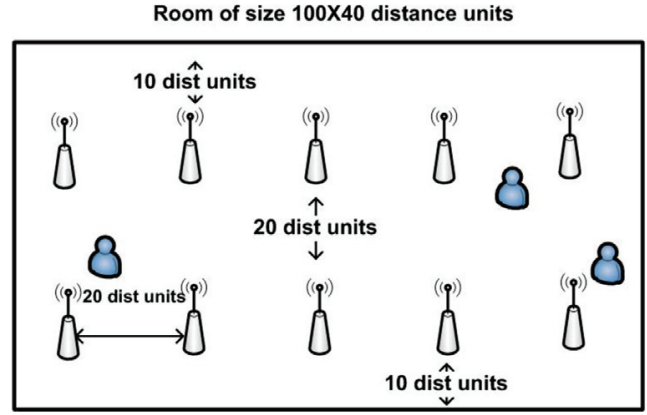

(b) Access Points

Figure 2: Simulation topology.

WiFi APs offer access to the Internet. Each AP is considered to belong to a distinct access network. In each network, the AP is backed-up by a single cache of size $c$, which provides connectivity to a single content provider as illustrated in Figure 2(a). We assume that the APs are deployed in an orthogonal room/area of $100 \mathrm{X} 40$ distance units. The APs are deployed in two rows (5 APs per row), where the rows are 20 distance units away and each row is 10 distance units away from the edge of the room. Each AP is also 20 distance units away from the neighboring APs of the same row. Figure 2(b) illustrates the considered AP topology.

Users enter the considered area from random directions and activate their $\mathrm{WiFi}$ interfaces at randomly selected locations. Upon activation users remain at their location until they switch off their WiFi interface. While activated a UE can only associate with APs that are within a radius of $R$ distance units. In order to take into consideration the characteristics of the network topology in this work we consider two distinct cases:

- Full coverage, where a sufficiently large $R$ value guarantees full observability of the existing networks (i.e., $R=100$ distance units in the topology of Figure 2(b)).
The purpose of this case is to isolate the impact of the proposed mechanism by ensuring uniform availability of network and storage (cache) resources across all UEs.

- Limited coverage, where a limited value of $R=30$ distance units results in UEs anticipating a subset of the available networks, and corresponding cache resources.

Content request model. While activated, UEs request content from a catalogue of size $C=10^{5}$ items. Content popularity follows a Zipf-like distribution of slope $s$. $U$ unique UE profiles are derived from the Zipf-like distribution. Each profile consists of a set of $u$ unique, uniformly randomly selected items from the content catalogue.

Simulation Scenarios. We consider a scenario where $\frac{N}{3}$ of the users initially visit the mall/lounge and associate to a randomly selected AP. This connectivity decision is information-agnostic and uniformly random, given the simplifying assumption of uniform wireless conditions. Upon association, each user generates content requests at a rate of $0.1 \mathrm{req} / \mathrm{sec}$. We let caches stabilize so as to observe the cache performance in the absence of any ICCON-related mechanism. Subsequently, an arrival-departure process starts, in which, at each step, one already connected user departs from the system, and a new one enters it. Each newly arrived UE now connects to the AP leading to the cache with the best fit against its profile. In order to focus on the potential benefits of the proposed approach, isolating the effects of the particular Bloom filter based profiling scheme, here, we calculate $f$ as the ratio of a, UE profile's content items found in the LFU-index of the corresponding cache (in the per session association mode). Between consecutive departure/arrival events we also let caches stabilize so as to observe the cache performance. The user interarrival process has a rate $\lambda$ and completes once all $\frac{2 N}{3}$ ICCON-supported UEs enter the system.

In this context, we vary key parameters of our setup so as to investigate their impact on the observed performance. Table 1 summarizes the considered parameters and defaults values.

\begin{tabular}{|l|l|}
\hline Parameter & Default Value \\
\hline Cache size $(c)$ & $5 \% C$ \\
\hline UE Profile size $(u)$ & $10 \% C$ \\
\hline Number of profiles $(U)$ & 50 \\
\hline Zipf slope $(s)$ & 0.8 \\
\hline User arrival rate (users/sec) $(\lambda)$ & 0.001 \\
\hline
\end{tabular}

Table 1: Evaluated parameters and their default values ( $C$ is the the size of the content catalogue). 


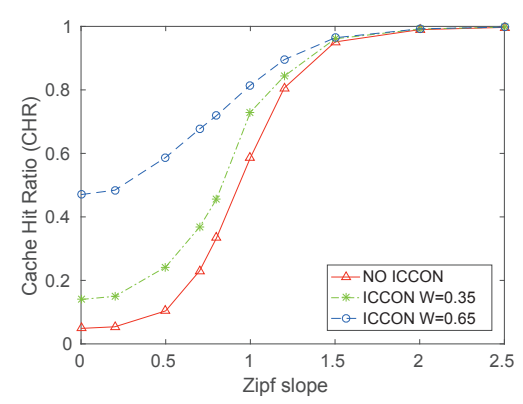

(a) Full coverage scenario

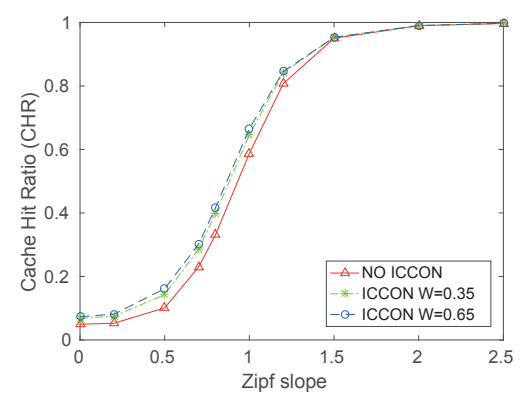

(b) Limited coverage scenario

Figure 3: CHR varying the Zipf slope for the per request association mode.

\subsection{Results}

We employ our simulation framework to evaluate the effects of the proposed mechanism on cache efficiency and the distribution of the UE load to the available networks.

\subsubsection{Cache efficiency}

Here we analyse the cache efficiency of the system as expressed by the Cache Hit Ratio (CHR) achieved. We evaluate ICCON using two different weights values $(W)$ in the fit function $F$ (see Eq. (2)), and compare it against an information-agnostic solution (NO ICCON in the plots) that only takes into account the load of the APs during the connectivity decision, i.e., the user associates with the least loaded AP within his coverage range (effectively equivalent to $W=0$ ).

Figures 3 and 4 depict the cache efficiency for the per request association mode (see Section 2.2). Figure 3 shows the average CHR for all the requests modifying the Zipf slope for the full and the limited coverage scenario. In both cases we can see a clear improvement of ICCON for all Zipf slope values between 0 and 1.5. In the full coverage scenario, this difference is more pronounced for small values of the Zipf slope using a $W=0.65$. We observe that the CHR is clearly improved when the weight in the fit function $F$ gives more importance to the UE profiling match than to the AP load. In the limited coverage, this improvement is less important since the small number of the AP avail-

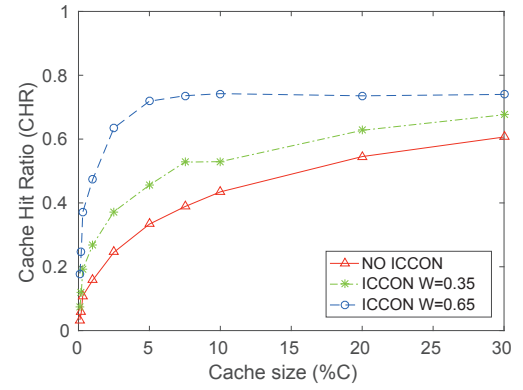

(a) Full coverage scenario

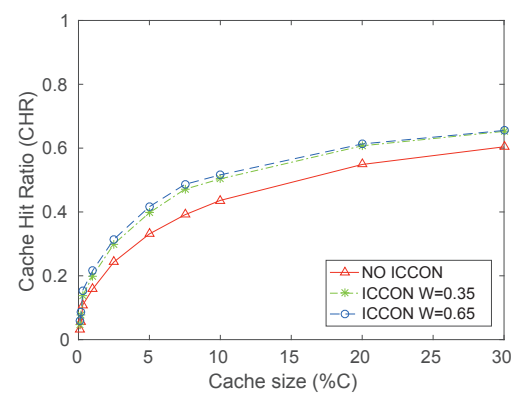

(b) Limited coverage scenario

Figure 4: CHR varying the cache size for the per request association mode.

\begin{tabular}{|c|c|c|}
\hline Scenario & $\mathbf{W = 0 . 3 5}$ & $\mathbf{W = 0 . 6 5}$ \\
\hline Full coverage & 36.66 & 115.46 \\
\hline $\begin{array}{c}\text { Limited } \\
\text { coverage }\end{array}$ & 20.02 & 25.73 \\
\hline
\end{tabular}

Table 2: ICCON CHR outperformance in \% using the default parameters with a per request association mode.

able within the range of a UE limits the possibilities of increasing the CHR exploring information-centric techniques.

Figure 4 shows the average CHR for all the requests varying the cache size ( $\%$ of the catalogue $C$ ) for the full and the limited coverage scenarios. Here we also observe that in both cases we can see a clear improvement of ICCON for any cache size. Again, this improvement is higher when the weight value $W$ is equal to 0.65 , however this difference is mostly clearly observed in the full coverage scenario, where the connectivity richness provides more opportunities to ICCON users to find the content requested in any of the available networks.

Table 2 summarizes the outperformance of ICCON using the weight values of $W=0.65$ and $W=0.35$ for the per request mode, and the default parameters indicated in Table 1 . The proposed mechanism yields up to $115.45 \%$ of CHR improvement over the information agnostic solution. This implies that our attempt to introduce information awareness at the link layer can dramatically offload the available networks and maximize 


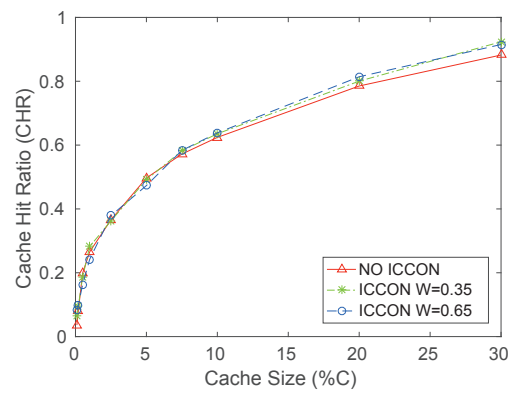

(a) Cache size

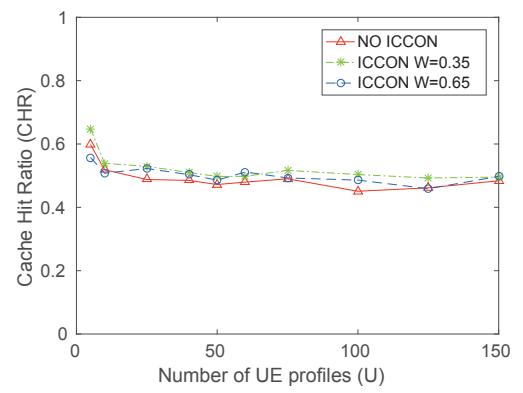

(b) Number of profiles

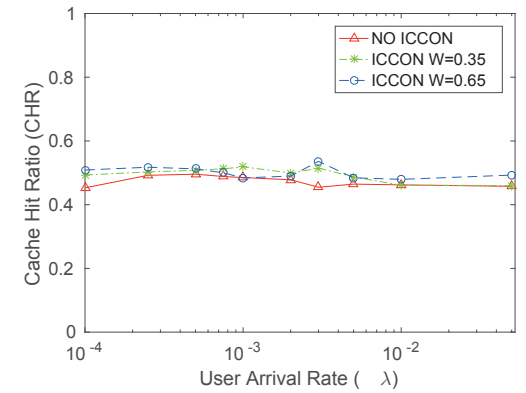

(c) Arrivals rate

Figure 5: CHR in the full coverage scenario for the per session association mode.

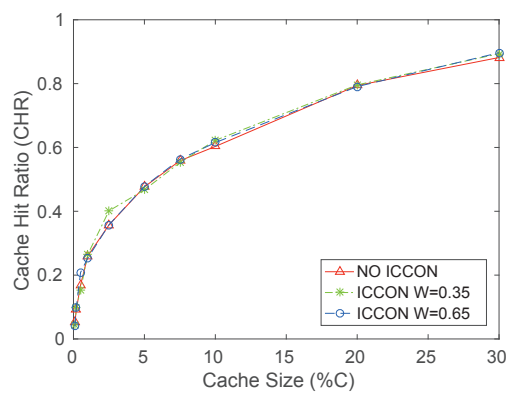

(a) Cache size

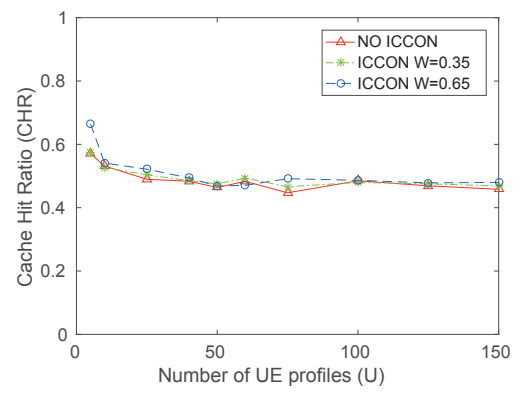

(b) Number of profiles

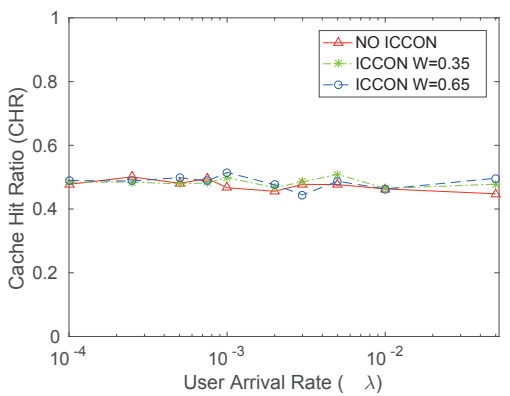

(c) Arrivals rate

Figure 6: CHR in the limited coverage scenario for the per session association mode.

the benefits of the inherent in-network capabilities of ICN.

In order to evaluate the CHR using the per session mode, we simulated the scenario detailed in the previous section and we analyzed the results modifying the cache size, the number of different user profiles and the user interarrival time.

In Figure 5 we observe the CHR in the full coverage scenario using the per session association mode. From Figure 5 we observe that ICCON outperforms the information-agnostic solution in all cases (even marginally). However, this improvement is not so evident as in the per request mode, since UE profile match does not depend on a unique item request and the cache of the network selected may have cached only part of the requested content.

Figure 5(a) shows the effect of the cache size on the observed CHR. ICCON improves CHR for any cache size, however we perceive a more pronounced difference for larger cache sizes. When the size of the caches is very small, the probability of finding the requested content decreases, therefore it is more difficult to have increased benefits as with the larger capacity sizes.

In Figure 5(b) we observe the CHR varying the number of user profiles in the simulation. For larger numbers of different UE profiles there is smaller correlation in the content requested by UEs, something that slightly decreases the overall benefits of the ICCON mechanism. In this cases, user interests are more di-

\begin{tabular}{|c|c|c|}
\hline Scenario & $\mathbf{W = 0 . 3 5}$ & $\mathbf{W = 0 . 6 5}$ \\
\hline Full coverage & 10.75 & 18.49 \\
\hline $\begin{array}{c}\text { Limited } \\
\text { coverage }\end{array}$ & 4.39 & 4.14 \\
\hline
\end{tabular}

Table 3: ICCON CHR outperformance in \% using the default parameters for the per session association mode.

verse leading to correspondingly diverse sets of content items in the caches. As a result, subsequent UE profile matches do not yield good fit (i.e., smaller values of the fit function in Eq. (2)).

Figure 5(c) illustrates CHR for different user interrarival rates. ICCON outperforms an information-agnostic solution in all cases, though with a marginal benefit. Evidently, the user interarrival rate does not really affect the cache efficiency of both ICCON and NO ICCON solutions.

In all cases, we see that, as expected, a larger weight value $(W=0.65)$ yields better CHR as it favours the selection of the most promising cache, against the load metric $l$ (see Section 3.4).

Similarly, Figures 6(a), 6(b) and 6(c) depict the observed CHR in the limited coverage scenario. In this case, we observe a similar performance of the ICCON approach over an information-agnostic solution. The performance perceived when varying the cache size, the number of user profiles and the user interarrival rate is 


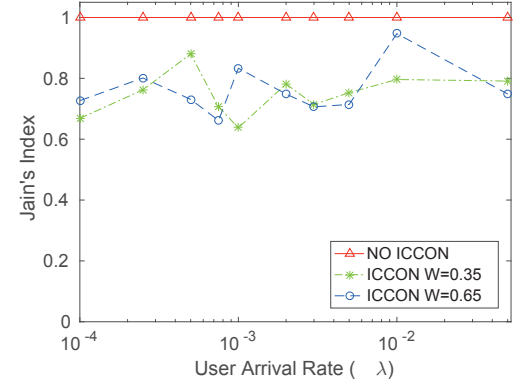

(a) Full coverage scenario

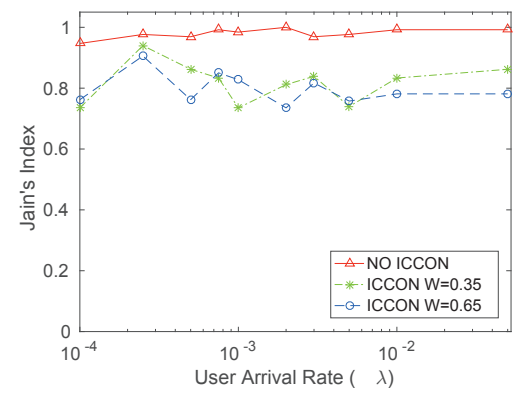

(b) Limited coverage scenario

Figure 7: AP load distribution varying the user interarrival rate.

almost the same as in the full coverage scenario, with both solutions performing slightly worse compared to the full coverage scenario, due to the limited connectivity options for each UE.

Table 3 summarizes the outperformance of ICCON using the weight values of $W=0.65$ and $W=0.35$ for the per session association mode, and the default parameters indicated in Table 1 . Though we notice a considerable lower benefit, compared to the per request association mode, ICCON still outperforms up to $\approx 19 \%$ an information-agnostic solution.

\subsubsection{Load}

The introduction of information-awareness in the network selection process promises an increase of the perceived CHR, as similar requests are headed to the same APs. This effectively means that ICCON favours the clustering of users with similar interests. However, this clustering behaviour, also affects the distribution of the overall workload (i.e., traffic, contention, etc.) in the entire set of APs and the corresponding networks. In order to get a better understanding of these effects, we also examine the distribution of UEs across the available APs. Given the uniform behavior of users, in terms of both mobility and content request rates, we believe this aspect adequately reflects our targeted aspects. In the following we employ Jain's fairness index [15] for the number of UEs associated at each AP. An index value of 1 denotes a uniform, equal distribution of UEs to the available APs, while the worst case value is $1 / M=0.1$, in our scenario.

Due to space limitations, in Figure 7 we depict the load fairness index for both the full and the limited coverage scenarios only for the case of different user arrival rates, whereas we report similar behaviour for the rest of the examined parameters. From Figure 7, we observe that when using an information-agnostic solution the clients are equally distributed among the APs (the index is 1) for the full coverage scenario. For the limited coverage scenario the client distribution is not totally equal, since UEs cannot connect to all APs, but the distribution is quite fair since the index is close to 1 . As expected, the ICCON solution results in an unbalanced load distribution (i.e., lower than 1), but when in the fit function we increase the weight of the APs load (see Section 3.4), the observed imbalance is rather limited (close to 0.65 in the worst case). This imbalance is more pronounced in the full coverage scenario, since UEs are presented with more connectivity options and hence more opportunities to cluster around APs.

\section{RELATED WORK}

As mentioned in Section3.4, vertical handover decisions and offloading mechanisms have been extensively studied in the past in an effort to identify the appropriate criteria and algorithms to assist in connectivity decisions for UEs [22, 2]. However, these approaches have focused on the conditions observed in the corresponding (wireless) network, overlooking the importance of information availability. On the other hand, some works in the past have considered the use of link-layer mechanisms to convey information to UEs. In [10], Chandra, et al. investigate mechanisms allowing UEs to retrieve information carried by 802.11 beacons. The conveyed information can be used also to guide network selection decisions. While this approach certainly contributes to the same goals as our work, it allows the delivery of only limited information to UEs. It focuses only on WiFi networks and has not been followed by a quantitative evaluation on the expected benefits. Wirtz, et al. in [21] propose the use of similar mechanisms to allow the direct delivery of information (e.g., content) to users. However, in the proposed approach an SSID is setup per content item request, leading to the allocation of wireless network resources for a single content item. On the contrary, our work foresees the exchange of information at the link layer only as an enabler for the preferred selection of a network, with actual data delivery taking place upon association. Recently, Schneider, et al. investigated the ability of CCN/NDN architecture to simultaneously use multiple access networks through a mobile device's multiple network interfaces [18]. We consider our work orthogonal and complementary to this approach, as we rather focus on the selection of 
the most promising access network available per network interface.

\section{CONCLUSIONS AND FUTURE WORK}

As the connectivity opportunities for mobile devices increases, the corresponding information availability in the networking environment becomes increasingly richer. However, current mobile device behaviour cannot capture this richness, being inherently tight to informationagnostic connectivity management methods. Though ICN has brought information-awareness in the forefront of networking, it also overlooks its importance in taking connectivity decisions. In this paper, we have built on this observation, making a first step towards the design and evaluation of an information-aware access network selection approach. Focusing on the case of WiFi offloading, we proposed mechanisms for the selection of APs (and corresponding access network), taking into account information-availability in the form of cached content. Our preliminary results show an increase of the perceived cache hit ration, in some cases by even up to $115 \%$, compared to an information-agnostic scheme.

However, we consider this work only as a first step in the investigation of information-awareness for connectivity management, as series of open research issues emerge. The use of Bloom filters for the creation of user and cache/network profiles, calls for further investigation, in terms of the Bloom filter configuration (i.e., size, number of hash functions) in view of the available cache sizes and the lifetime of content items in the cache. At the same time, the overheads associated with the exchange of profiles call for a close investigation. Alternative approaches inspired by previous work on set reconciliation methods are also included in our future work plans [7]. At the same time, the effect of different network and cache topologies also calls for a closer investigation, e.g., hierarchical cache topologies, caches shared by different APs, etc.

Taking a step further, we believe that the proposed concept presents particular interest in different networking environments. Example cases include Hetnets, where information availability can be taken into account in cell selection, as well as devise-to-device (D2D) communications, where pairwise or even group communication can be triggered and shaped by the information available, e.g., WiFi Direct Group formation. Moreover, information availability can be revisited in the context of in-network services and/or applications, calling for the investigation of different profiling and matching mechanisms.

\section{REFERENCES}

[1] 3GPP. Technical Specification Group Services and System Aspects; Architecture enhancements for non-3GPP accesses. TS 23.402 V13.3.0, 3rd Generation Partnership Project (3GPP), Sept. 2015.
[2] A. Aijaz, H. Aghvami, and M. Amani. A survey on mobile data offloading: technical and business perspectives. IEEE Wireless Communications, 20(2):104-112, April 2013.

[3] A. Akella, G. Judd, S. Seshan, and P. Steenkiste. Self-management in chaotic wireless deployments. In Proceedings of the 11th Annual International Conference on Mobile Computing and Networking, MobiCom '05, pages 185-199. New York, NY, USA, 2005. ACM.

[4] J. G. Andrews. Seven ways that hetnets are a cellular paradigm shift. IEEE Communications Magazine, 51(3):136-144, March 2013.

[5] B. H. Bloom. Space/time trade-offs in hash coding with allowable errors. Commun. ACM, 13(7):422-426, July 1970.

[6] F. Bonomi, R. Milito, J. Zhu, and S. Addepalli. Fog computing and its role in the internet of things. In Proceedings of the First Edition of the MCC Workshop on Mobile Cloud Computing, MCC '12, pages 13-16, New York, NY, USA, 2012. ACM.

[7] A. Z. Broder. On the resemblance and containment of documents. In Compression and Complexity of Sequences 1997. Proceedings, pages 21-29, Jun 1997.

[8] D. Camps-Mur, E. Garcia-Villegas, E. Lopez-Aguilera, P. Loureiro, P. Lambert, and A. Raissinia. Enabling always on service discovery: Wifi neighbor awareness networking. IEEE Wireless Communications, 22(2):118-125, April 2015.

[9] Z. Cao, J. Fitschen, and P. Papadimitriou. Freesurf: Application-centric wireless access with sdn. SIGCOMM Comput. Commun. Rev., 45(4):357-358, Aug. 2015.

[10] R. Chandra, J. Padhye, L. Ravindranath, and A. Wolman. Beacon-stuffing: Wi-fi without associations. In Mobile Computing Systems and Applications, 2007. HotMobile 2007. Eighth IEEE Workshop on, pages 53-57, March 2007.

[11] D. Evans. The Internet of Everything: How More Relevant and Valuable Connections Will Change the World. White Paper, February 2012

[12] IEEE Standard 802.11u. IEEE Standard for Information Technology-Telecommunications and information exchange between systems-Local and Metropolitan networks-specific requirements-Part II: Wireless LAN Medium Access Control (MAC) and Physical Layer (PHY) specifications: Amendment 9: Interworking with External Networks. pages 1-208, Feb 2011

[13] InterDigital. Strategies for Effective Wi-Fi Offload. Technical Report White Paper, InterDigital, 2015.

[14] V. Jacobson, D. K. Smetters, J. D. Thornton, M. F. Plass, N. H. Briggs, and R. L. Braynard. Networking named content. In Proceedings of the 5th International Conference on Emerging Networking Experiments and Technologies, CoNEXT'09, pages 1-12, New York, NY, USA, 2009. ACM.

[15] R. Jain, D. Chiu, and W. Hawe. A quantitative measure of fairness and discrimination for resource allocation in shared computer systems. CoRR, cs.NI/9809099, 1998.

[16] H. Lee and A. Nakao. User-assisted in-network caching in information-centric networking. Computer Networks, 57(16):3142 - 3153, 2013. Information Centric Networking.

[17] D. Rossi and G. Rossini. Caching performance of content centric networks under multi-path routing (and more). TR, Telecom ParisTech, 2011.

[18] K. M. Schneider and U. R. Krieger. Beyond network selection: Exploiting access network heterogeneity with named data networking. In Proceedings of the 2Nd International Conference on Information-Centric Networking, ICN '15, pages 137-146, New York, NY, USA, 2015. ACM.

[19] V. Sourlas, L. Tassiulas, I. Psaras, and G. Pavlou. Information resilience through user-assisted caching in disruptive content-centric networks. In IFIP Networking Conference (IFIP Networking), 2015, pages 1-9, May 2015.

[20] A. Varga. OMNeT++ Simulator Home Page. http://www.omnetpp.org.

[21] H. Wirtz, M. Ceriotti, B. Grap, and K. Wehrle. Pervasive content-centric wireless networking. In World of Wireless, Mobile and Multimedia Networks (WoWMoM), 2014 IEEE 15th International Symposium on a, pages 1-9, June 2014.

[22] X. Yan, Y. A. ekerciolu, and S. Narayanan. A survey of vertical handover decision algorithms in fourth generation heterogeneous wireless networks. Computer Networks, 54(11):1848 - 1863, 2010. 